$\mathrm{PSU} / \mathrm{TH} / 102$

March 23, 1993

\title{
Fragmentation of Transversely Polarized Quarks Probed in Transverse Momentum Distributions
}

\author{
John Collins \\ Physics Department, Penn State University, \\ University Park, PA 16802, U.S.A.
}

\begin{abstract}
It is shown that the azimuthal dependence of the distribution of hadrons in a quark jet is a probe of the transverse spin of the quark initiating the jet. This results in a new spin-dependent fragmentation function that acts at the twist-2 level. One example of a process where it contributes is semi-inclusive deeply inelastic scattering with a transversely polarized nucleon target but with an unpolarized electron beam. This process is treated in detail. Another process is the cross section for singly polarized hadron-hadron scattering when two high $p_{\perp}$ hadrons are measured in the final state and are close to back-to-back in azimuth. The new fragmentation function is sensitive to the coupling of the fragmentation process to (spontaneous) chiral symmetry breaking.
\end{abstract}

\section{Introduction}

An important challenge to QCD theorists is to devise methods of measuring the polarization state of a parton coming out of a hard scattering. In the case of longitudinal polarization for the parton, Nachtmann [1] showed how a certain three-particle correlation within a jet could be used. He suggested several processes where it could be measured, in particular deep inelastic neutrino scattering. Later, Dalitz, Goldstein and Marshall [2] and Einhorn [3] showed how to probe the helicity of a heavy quark.

Recently Efremov, Mankiewicz and Törnqvist [4] rediscovered the Nachtmann idea, which they called the 'handedness' of a jet, and they showed how to measure it in $e^{+} e^{-}$annihilation. They also considered the possibility of probing the transverse polarization of quarks. This idea was independently discovered in [5].

In this paper, I will present another technique sensitive to the transverse polarization of a quark. One measures the single particle distribution as a function of transverse momentum, in a process such as semi-inclusive deeply inelastic scattering, where the jet axis can be precisely defined. The initial-state hadron is transversely polarized, and the hard scattering 
provides a spin transfer to the final state. There should be a spin asymmetry in the azimuthal distribution of the outgoing hadron about the jet axis.

One could argue that the large number of hadrons produced in a jet would completely dilute this asymmetry. However, it is known that for flavor quantum numbers, the leading particle in a jet is correlated in flavor with the quark initiating the jet [6]. Moreover, much of the hadron production results from the emission of gluons at small $z$; this preserves the helicity state of the quark, and it is the quark that is responsible for the leading hadrons.

Measurements like this probe three quantities, none of which have yet been measured: the transverse spin of a quark in a transversely polarized hadron, the transverse spin dependence of hard scattering, and the spin-dependence of fragmentation. The difference between distributions for the transverse spin and for the helicity of a quark in a hadron is a measure of relativistic effects in the hadron's wave function [7]. Transverse spin dependence of the fragmentation involves a helicity flip for the quark, and is therefore sensitive not only to the spin dependence of fragmentation in general but to the presence of (non-perturbative) chiral symmetry breaking.

For the single-particle measurement, one must have a process where there is a definite axis or plane with respect to which transverse momentum of an outgoing particle can be defined. Two obvious examples are:

- Semi-inclusive deeply inelastic scattering, $e+p^{\uparrow} \rightarrow e+H+X$, when the kinematic variables $x_{\mathrm{Bj}}$ and $z$ are not small, and the transverse momentum of the observed hadron (relative to the plane of the initial proton and the two electrons) is moderate. This is the process that I will treat in detail in this paper.

- $p+p^{\uparrow} \rightarrow H_{1}+H_{2}+X$, when the two observed final-state hadrons have large $p_{T}$ relative to the axis of the initial-state particles and are nearly back-to-back in azimuth about that axis.

Here, $p^{\uparrow}$ denotes a transversely polarized hadron. Undoubtedly, other similar observables can be easily devised. It is also standard to define a jet axis by calorimetric measurements of the jet for example. In that case one can measure the azimuthal distribution of the leading pions about the axis. This probably suffers from lower precision in the definition of the axis.

In the proton-proton case, if one makes measurements at sufficiently large center-ofmass rapidity, then one can have the collision of a small $x$ gluon and a moderately large $x$ valence quark. This allows one to take advantage of the large number of small $x$ gluons and the expected large polarization of the valence quarks. This would give both a large spin asymmetry and a large cross section.

Both the single-particle measurement suggested in this paper and the two-particle measurement suggested in $[4,5]$ are twist-2: that is, the spin asymmetries are not suppressed by a power of the large momentum scale $Q$ in the hard scattering. This is important because of the paucity of useful twist-2 asymmetries involving transverse spin. The problem is that transverse spin asymmetries involve off-diagonal elements in the helicity density matrices of the quarks, and that the hard scattering preserves quark helicity. (It is quarks, not gluons 
that are relevant here [8].) Thus to get a twist-2 asymmetry, the non-perturbative part of the process (consisting of the parton distribution and fragmentation functions) must contain an even number of factors that flip quark helicity.

The obvious measurements that are sensitive to transverse spin at the twist-2 level involve collisions of two polarized hadrons, and to get a large asymmetry one wants to be in the valence region for the quarks entering the hard scattering. The two standard processes are jet production and the Drell-Yan process. But jet production has a numerically small asymmetry [9] in the hard scattering. The Drell-Yan process, with a large asymmetry in the hard scattering [10], needs a polarized antiproton beam with good luminosity to make it a valence process with a reasonable event rate.

In contrast, the spin asymmetries discussed in this paper, and those in $[4,5]$ have their second measured spin in the final state instead of the initial state. It is interesting that these fragmentation measurements are easier for transverse polarization than for longitudinal. A transverse polarization can be probed by measuring two suitable momentum vectors (e.g., two particles in a jet, or one particle and a jet axis). But a longitudinal polarization requires three vectors, to define a handedness - see [1,4], and also the work of Carlitz and Willey [11].

The new spin dependent fragmentation effect that is the subject of this paper gives a leading-twist contribution only to processes that are sensitive to intrinsic transverse momentum. But it may also have consequences for other processes. For example, consider the inclusive cross section for production of a high $p_{\perp}$ hadron in hadron-hadron collisions. When only one of the initial hadrons is transversely polarized, QCD predicts that the resulting spin asymmetry is of twist-3. There is a notorious contrast between this prediction and the large asymmetry actually measured [12]. The experiments are admittedly at rather modest energies (fixed target with beams of up to $200 \mathrm{GeV}$ ). Methods for treating twist-3 effects are currently begin developed $[7,13,14]$.

In that process, there is effectively an average over intrinsic transverse momentum, and in particular over its azimuthal angle about the jet axis. Therefore the spin-dependence of the fragmentation that is treated in the present paper gets canceled, at the leading twist level. But the cross section is a steeply falling function of the hadron's $p_{\perp}$, so that the azimuthal average need not work very well. The result could be a numerically large twist-3 contribution in the fragmentation. The authors of [14] have not yet treated twist 3 effects in fragmentation.

The layout of this paper is as follows. I define the fragmentation and distribution functions in Sec. 2. Then in Sec. 3 I show how these get used in deeply inelastic scattering, and in Sec. 4 I explain the polarization sensitive measurements that can be made; that section is perhaps the one of most use to experimentalists. In Sec. 5, I make a few remarks about hadron-hadron scattering. Then I exhibit some simple model calculations in Sec. 6 to show that the fragmentation asymmetry I propose does not violate any fundamental principles. I summarize my conclusions in Sec. 7. 


\section{Spin-Dependence of Parton Distribution and Fragmentation Functions}

In this section, I will first review the formal definitions of the parton distribution and fragmentation functions when there is a measured transverse momentum. Then I will show how to extend the definitions to treat nontrivial polarization. These quantities will get used in factorization formulae for the cross section, as explained in later sections.

In the usual factorization theorems $[15,16]$, one works with parton densities integrated over transverse momentum. But when one has a cross section with a measured small transverse momentum variable, one must use the unintegrated distributions. In QCD, there are some interesting effects associated with Sudakov form factors, that make the resulting factorization theorems quite nontrivial [17]. The Sudakov effects are spin-independent, and we will not bother making them explicit here, since our purpose is to examine the novel effects associated with polarization. However when the energy of the experiment increases, the Sudakov effects will dilute our asymmetries by smearing out the transverse momentum distributions.

We will denote the unpolarized distribution and fragmentation functions by $f_{i / H}$ and $D_{H / i}$ respectively, when transverse momentum is integrated over. To denote the corresponding quantities with unintegrated transverse momentum, we will use the same symbols, but with a hat over them: $\hat{f}_{i / H}$ and $\hat{D}_{H / i}$.

\subsection{Parton Distribution Functions}

We define parton distribution functions by formulae motivated by light-front quantization. These quantities are precisely those that occur in the factorization theorems $[18,19,20]$.

It follows from the parity and time-reversal invariance of QCD that the number density of quarks is independent of the spin state of the initial hadron, so that we have

$$
\hat{f}_{a / A}\left(x,\left|k_{\perp}\right|\right) \equiv \int \frac{\mathrm{d} y^{-} \mathrm{d}^{2} y_{\perp}}{(2 \pi)^{3}} \mathrm{e}^{-i x p^{+} y^{-}+i k_{\perp} \cdot y_{\perp}}\left\langle p\left|\bar{\psi}_{i}\left(0, y^{-}, y_{\perp}\right) \frac{\gamma^{+}}{2} \psi_{i}(0)\right| p\right\rangle .
$$

We have ignored here the subtleties needed to make this a gauge invariant definition: an appropriate path ordered exponential of the gluon field is needed [18]. The coordinate frame in which this definition is applied is one in which the hadron $|p\rangle$ has zero transverse momentum: $p_{\perp}=0$.

Sivers [21] suggested that the $k_{\perp}$ distribution of the quark could have an azimuthal asymmetry when the initial hadron has transverse polarization. However, such an asymmetry is prohibited because QCD is time-reversal invariant. This is shown in the appendix.

As explained in $[10,22]$, we must consider the quark (or gluon) $a$ to be equipped with a helicity density matrix. Since QCD is invariant under parity and time reversal, the density matrix for a quark differs from unity only if the initial hadron $A$ is itself polarized. Then 
the transverse spin asymmetry of a quark is defined by:

$$
\begin{aligned}
s_{\perp}^{\mu} \hat{\Delta}_{T a / A} \hat{f}_{a / A}\left(x, k_{\perp}\right) & \equiv s_{\perp}^{\mu} \hat{f}_{T a / A}\left(x, k_{\perp}\right) \\
& \equiv \int \frac{\mathrm{d} y^{-} \mathrm{d}^{2} y_{\perp}}{(2 \pi)^{3}} \mathrm{e}^{-i x p^{+} y^{-}+i k_{\perp} \cdot y_{\perp}}\left\langle p\left|\bar{\psi}_{a}\left(0, y^{-}, y_{\perp}\right) \frac{\gamma^{+} \gamma_{\perp}^{\mu} \gamma_{5}}{2} \psi_{a}(0)\right| p\right\rangle,
\end{aligned}
$$

where $s_{\perp}^{\mu}$ is the transverse part of the spin vector of the initial hadron, normalized so that its maximum size is unity: $\left|s_{\perp}\right| \leq 1$. In eq. (2), I have used the notation of [22], where definitions are given for the case that the transverse momentum is integrated over; the definitions given for that case given by Jaffe and Ji [7] differ only in notation. I have defined $\hat{\Delta}_{T}$ to mean the ratio of quark polarization to hadron polarization; it is a kind of asymmetry or spin transfer function, and in general will depend on $x$ and $k_{\perp}$. Then $\hat{f}_{T}$ (with a subscript $T$ ) means the parton distribution weighted by the transverse spin asymmetry.

Similar definitions can be given for the distribution of gluons. It would be interesting to work out the details. For the deep-inelastic process treated in this paper, we will see that we will not need the definitions for gluons.

One can also write helicity asymmetries. But we will not need them in this paper, because we will work with fragmentation observables that are not sensitive to quark helicity.

\subsection{Fragmentation Functions}

Fragmentation functions with transverse momentum are defined in a similar fashion to the parton distribution functions. An important difference is that the observed final-state hadron, assumed to be a pion, has no polarization, so that a spin-transfer function analogous to eq. (2) does not exist. On the other hand we can no longer apply the generalization of the time-reversal argument that prevented an azimuthal asymmetry in the number density, as we will explain.

The unpolarized fragmentation function, to find a hadron $H$ in the decay products of a quark of flavor $a$ is [18]

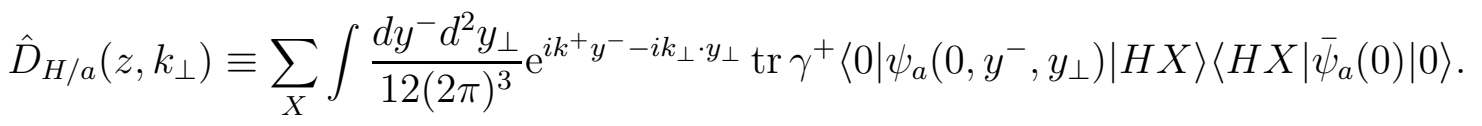

The factor of $1 / 12$ is the product of a factor $1 / 2$ which occurs with all these definitions for fermions and a factor $1 / 6$ for an average over the spin and colors states of the quark. The sum over $X$ is over all final states containing the chosen hadron.

This definition is easily generalized to give the transverse spin dependence $\Delta \hat{D}$ of the distribution of hadrons in a polarized quark of transverse spin $s_{\perp}^{\mu}$ :

$$
\begin{aligned}
\Delta \hat{D}_{H / a}\left(z, k_{\perp}, s_{\perp}\right) & \equiv \\
& \sum_{X} \int \frac{d y^{-} d^{2} y_{\perp}}{12(2 \pi)^{3}} \mathrm{e}^{i k^{+} y^{-}-i k_{\perp} \cdot y_{\perp}} \operatorname{tr} \gamma^{+} \gamma_{5} \gamma_{\perp} \cdot s_{\perp}\left\langle 0\left|\psi_{a}\left(0, y^{-}, y_{\perp}\right)\right| H X\right\rangle\left\langle H X\left|\bar{\psi}_{a}(0)\right| 0\right\rangle .
\end{aligned}
$$


This is permitted to depend on the spin through the following scalar quantity:

$$
\epsilon_{\kappa \lambda \mu \nu} s_{\perp}^{\kappa} k_{\perp}^{\lambda} p_{H}^{\mu} n^{\nu}
$$

where $n^{\mu} \equiv \delta_{-}^{\mu}$ is the vector that is used to define the light-front momentum fraction $z$ : $z \equiv p_{H}^{+} / k^{+}=p_{H} \cdot n / k \cdot n$, with $k^{\mu}$ being the momentum of the quark. (Note that the above definitions imply that $k^{-}$is integrated over, while $k_{\perp}$ is the transverse momentum of the quark relative to the hadron $H$.)

There is some disagreement in the literature on the normalization of the fragmentation functions. The definitions given above are the most convenient for theoretical analyses since the bilocal vertex for the quark depends only on momenta defined at that vertex. However, a probability interpretation is most naturally given in terms of the transverse momentum of the hadron relative to the quark, which is $p_{\perp}=-z k_{\perp}$. Then the probability density in $z$ and $p_{\perp}$ is $z^{-1} \hat{D}\left(z,-z p_{\perp}\right)$. As for the density integrated over transverse momentum, i.e., $D(z) \equiv \int d^{2} k_{\perp} \hat{D}\left(z, k_{\perp}\right)$, the probability density is $z D(z)$. (This implies that the momentum sum rule is $\sum_{H} \int_{0}^{1} z^{2} D_{H / a}(z) d z=1$.)

Now time reversal invariance prohibits an asymmetry proportional to (5), unless there are nontrivial phases from final state interactions; but such phases can surely exist in a strong interaction fragmentation. Another way of saying this comes from observing that a combination of time reversal and parity invariance transforms out-states to in-states of the same momentum content. (Compare the arguments given in the appendix for the distribution functions.) If the in-states were the same as the out-states, then we could show that the asymmetry proportional to (5) is zero. For strongly nonperturbative interactions, such as are involved in hadronization, this is surely not true. Similar arguments have been applied to polarized pion-nucleon scattering [23].

\section{Semi-Inclusive Deeply Inelastic Scattering}

\subsection{Kinematics}

Semi-inclusive deeply inelastic scattering is the process $e+A \rightarrow e^{\prime}+B+X$ where $A$ is some arbitrary initial hadronic state of momentum $p_{A}^{\mu}$, and $B$ is an observed hadron in the final state of momentum $p_{B}^{\mu}$. The momentum transfer from the lepton system is $q^{\mu}$, as usual, and we define the masses of hadrons $A$ and $B$ to be $M_{A}$ and $M_{B}$.

In the absence of polarization, there are four Lorentz invariant variables for the hadronic system, viz., $q^{2}, p_{A} \cdot q, p_{B} \cdot q$, and $p_{A} \cdot p_{B}$. A useful set of variables was defined by Meng, Olness and Soper [24]. The two variables not involving the final-state hadron $B$ are, as usual,

$$
x_{\mathrm{Bj}} \equiv \frac{Q^{2}}{2 p_{A} \cdot q},
$$

and

$$
Q \equiv \sqrt{-q^{2}}
$$




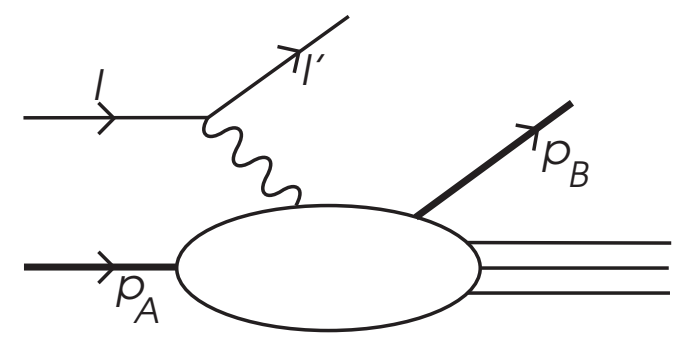

Fig. 1. Kinematics of semi-inclusive deeply inelastic scattering.

Then as in [25] one defines

$$
z \equiv \frac{p_{A} \cdot p_{B}}{p_{A} \cdot q} .
$$

Finally, Meng et al. define $q_{\perp}^{\mu}$ as the transverse momentum of $q^{\mu}$, in a frame where $p_{A}^{\mu}$ and $p_{B}^{\mu}$ have zero transverse momentum. Up to corrections that are irrelevant in the Bjorken limit, this means that

$$
q_{\perp}^{\mu}=q^{\mu}+\bar{x}_{A} p_{A}^{\mu}-\frac{1}{z} p_{B}^{\mu},
$$

where $\bar{x}_{A} \equiv-p_{B} \cdot q / p_{A} \cdot p_{B}=x_{\mathrm{Bj}}\left(1-\left|q_{\perp}^{2}\right| / Q^{2}\right)$.

The deep inelastic region is where $Q$ is made large, with $x_{\mathrm{Bj}}$ and $z$ held fixed and not close to their endpoints 0 and 1 . We will always assume in this paper that the scattering is taken to lowest order in QED, with a single photon being exchanged between the lepton and the hadronic system, Fig. 1.

The reason for defining the variables $x_{\mathrm{Bj}}, z$ and $q_{\perp}$ is that they have a simple interpretation in the parton model. There, it is assumed that the dominant contributions to the cross section have the form of Fig. 2. The virtual photon interacts in Born approximation with a single quark, which is close to its mass shell and which has low transverse momentum on the scale $Q$. Then when hadron $B$ is part of the 'current quark jet' produced in the hard scattering, $z$ has the interpretation of the fraction of the jet's momentum that is carried by the hadron. As usual, $x_{\mathrm{Bj}}$ has the interpretation of the fraction of the momentum of the incoming hadron $A$ that is carried by the parton that enters the hard scattering.

To treat intrinsic transverse momentum for the initial state and for the fragmentation, 


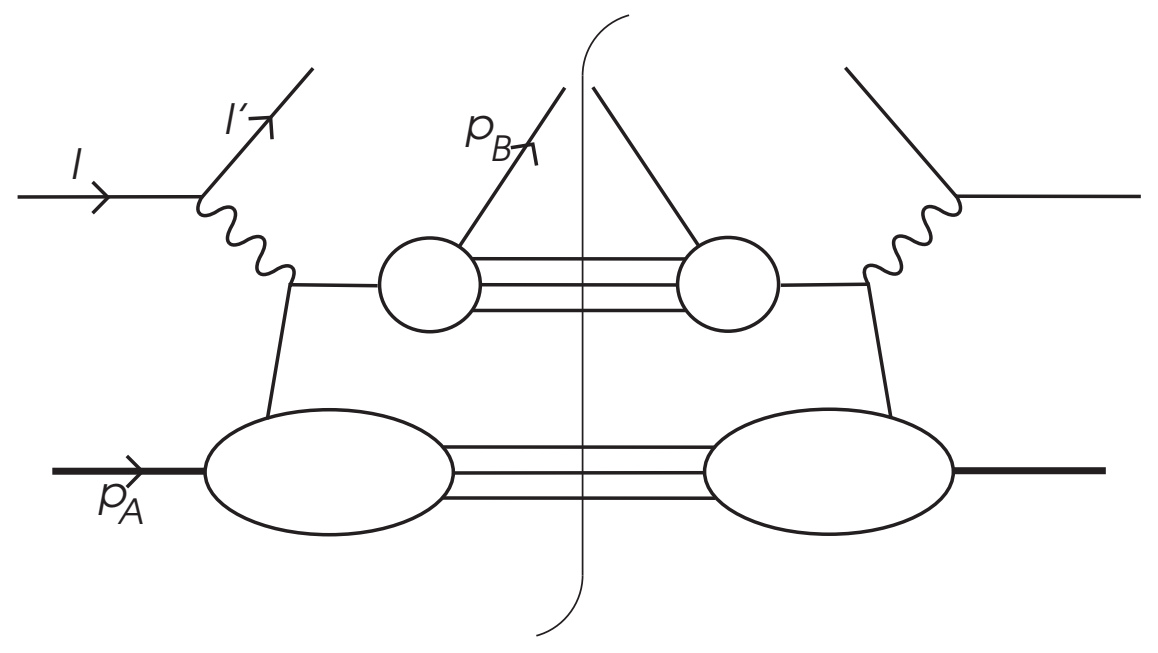

Fig. 2. Parton model for semi-inclusive deeply inelastic scattering. 
we need a suitable frame in which to define them. First we define what we will call the 'parton model jet axis':

$$
p_{J}^{\mu} \equiv q^{\mu}+x_{\mathrm{Bj}} p_{A}^{\mu}
$$

This would be the jet momentum if there were no intrinsic transverse momentum. Even in the presence of transverse momentum, the definition (10) gives a convenient axis with respect to which to define the transverse momentum of the outgoing hadron $B$.

As stated above, $q_{\perp}$ is the transverse momentum of the virtual photon in a frame in which the two hadrons $A$ and $B$ have zero transverse momentum. This is perhaps the simplest frame in which to derive the factorization theorem at low transverse momentum. But when we consider the azimuthal distribution of the outgoing hadron $B$ it will be more intuitive to consider the transverse momentum of $B$ relative to the parton model jet axis. This we can define by

$$
p_{B T}^{\mu} \equiv p_{B}^{\mu}-\frac{1}{z} p_{J}^{\mu}
$$

Note that to avoid some possible confusion we use the subscript ${ }_{T}$ here rather than $\perp_{\perp}$. This

is to emphasize that $p_{B T}^{\mu}$ is a four vector rather than the transverse two-component vector defined in a particular frame: the frame in which we define the fragmentation function will have zero transverse momentum for $B$.

Up to corrections of relative order $q_{\perp}^{2} / Q^{2}$, the transverse momentum $p_{B T}^{\mu}$ is $-z q_{\perp}^{\mu}$.

\subsection{FACTORIZATION Without INTRINSiC TRANSVERSE MOMENTUM}

In QCD, there is a standard factorization theorem:

$$
E^{\prime} E_{B} \frac{d \sigma}{d^{3} l^{\prime} d^{3} p_{B}}=\sum_{a, b} \int d \xi \int \frac{d \zeta}{\zeta} f_{a / A}(\xi) \text { (Hard scattering) } D_{B / b}(\zeta)
$$

which is correct up to power-law corrections. When the hard scattering factor is replaced by its lowest order approximation, we regain the parton model. In normalization the hard scattering function is the invariant cross section for lepton-parton scattering with the outgoing parton momentum set to $p_{B} / \zeta$.

This factorization theorem represents the asymptotics of the cross section when $Q$ gets large but the dimensionless ratios $x_{\mathrm{Bj}}, z$ are held fixed, and $q_{\perp} / Q$ is either held fixed or integrated over. This has the unfortunate implication that the factorization theorem fails to give a useful result for the distribution in $q_{\perp}$ when $q_{\perp} \ll Q$. However, this is exactly where the cross section is biggest. Indeed, in lowest order, the hard scattering function has a factor $\delta^{(2)}\left(q_{\perp}\right)$, while in higher order the hard scattering function has terms that diverge like $1 / q_{\perp}^{2}$ times logarithms when $q_{\perp} \rightarrow 0$.

A convenient way of formulating these statements is to state that the factorization theorem eq. (12) must be interpreted in the sense of distribution theory [26]. The theorem applies when the cross section is integrated with a smooth test function $\phi\left(x_{\mathrm{Bj}}, z, q_{\perp} / Q\right)$. In the derivation $[15,16,22]$, one applies to the hard scattering the approximation that the transverse momentum of the incoming parton can be neglected with respect to the transverse 
momentum generated in the scattering, and one also neglects the transverse momentum generated in the fragmentation. (Note that to the extent that these transverse momenta are not negligible, but are of order $Q$, the errors in the approximations are compensated by a correct treatment of higher order corrections to the hard scattering.)

\subsection{FACTORIZATION WITH INTRINSIC TRANSVERSE MOMENTUM BUT NO POLARIZATION}

To gain information on the $q_{\perp}$ dependence at small $q_{\perp}$, we must derive a more powerful theorem that involves 'intrinsic transverse momentum' in both the distribution and the fragmentation functions. Such a theorem was derived for the Drell-Yan process and for the two-particle-inclusive cross section in $e^{+} e^{-}$annihilation [27,17]. A similar theorem should apply here. An obvious ansatz is

$$
\begin{aligned}
E^{\prime} E_{B} \frac{d \sigma}{d^{3} l^{\prime} d^{3} p_{B}}= & \sum_{a} \int d \xi \int \frac{d \zeta}{\zeta} \int d^{2} k_{a \perp} \int d^{2} k_{b \perp} \hat{f}_{a / A}\left(\xi, k_{a \perp}\right) E^{\prime} E_{k_{b}} \frac{d \hat{\sigma}}{d^{3} l^{\prime} d^{3} k_{b}} \hat{D}_{B / a}\left(\zeta, k_{b \perp}\right) \\
& +Y\left(x_{\mathrm{Bj}}, Q, z, q_{\perp} / Q\right) .
\end{aligned}
$$

In this formula $\hat{\sigma}$ represents the short distance part of elastic lepton-quark scattering. It contains a delta-function for momentum conservation. The sum over $a$ is over all flavors of quark and antiquark.

The first term on the right of eq. (13) dominates when $q_{\perp} \ll Q$. The second term, $Y$, is a correction term that enables eq. (13) to reproduce the ordinary factorization theorem eq. (12) at large transverse momentum, just as in the Drell-Yan case [17]. The $Y$ term has the general form of the basic factorization theorem eq. (12), except that the low $q_{\perp}$ asymptote is subtracted from the hard scattering function.

The function $\hat{f}_{a / A}$ defined earlier gives the intrinsic transverse momentum dependence of

partons in the initial state hadron. Similarly, $\hat{D}_{B / a}$ gives the distribution of hadrons in a parton, with $k_{b \perp}$ being the transverse momentum of the parton relative to the hadron.

Just as in $[27,17]$, the hard scattering factor in the first term in eq. (13) can only be a $2 \rightarrow 2$ process. Hence the fractional momenta of the incoming quark from hadron $A$ and of hadron $B$ in the outgoing quark are forced to be $x_{\mathrm{Bj}}$ and $z$.

After integrating out the delta-function in $\hat{\sigma}$ we obtain

$$
\begin{aligned}
E^{\prime} E_{B} \frac{d \sigma}{d^{3} l^{\prime} d^{3} p_{B}}= & \frac{4 x_{\mathrm{Bj}}}{Q^{2}} \sum_{a} \int d^{2} k_{a \perp} \hat{f}_{a / A}\left(x_{\mathrm{Bj}}, k_{a \perp}\right) \frac{d \hat{\sigma}}{d \Omega} \hat{D}_{B / a}\left(z, k_{a \perp}+q_{\perp}\right) \\
& +Y\left(x_{\mathrm{Bj}}, Q, z, q_{\perp} / Q\right) .
\end{aligned}
$$

The picture that goes with these results is Fig. 2. All that we have done is to take account of the transverse momentum of the quarks relative to the measured initial- and final-state hadrons. This intrinsic transverse momentum has the effect of smearing out the delta function of $q_{\perp}$ that we remarked on earlier. The only generalization needed compared with the parton model is that the hard scattering can contain higher order virtual corrections. 
In the absence of gauge bosons in the strong interactions, this formula in the exact form given in eq. (13) is a theorem, that can be proved as in the Drell-Yan case [19].

The spin-1 gluons of QCD modify the theorem, by causing Sudakov form-factor effects. We expect that a proof can be given just as in the Drell-Yan case [17]. The effect is to broaden the transverse momentum distribution as $Q$ increases, but in a spin-independent way: the broadening is due to recoil against the transverse momentum of soft gluon emission. This will have the effect of diluting the spin asymmetry we will discuss next.

\subsection{FACTORIZATION WITH INTRINSIC TRANSVERSE MOMENTUM AND POLARIZATION}

We now explain factorization for the semi-inclusive deep inelastic cross section when the incoming hadron $A$ is transversely polarized but the lepton remains unpolarized. (It is left as an exercise to treat the most general case.) The factorization theorems, eq. (12) and eq. (14), continue to apply when we include polarization for the incoming hadron, but with the insertion of helicity density matrices for in and out quarks; this is a simple generalization of the results in $[10,22]$.

The cross section will be linear in the transversity $s_{\perp}^{\mu}$ of the hadron (and also linear in its helicity $\lambda$ ). Because transverse spin for a spin- $\frac{1}{2}$ particle corresponds to off diagonal terms in the helicity density matrix, the other primary constraint comes from quark helicity conservation in the hard scattering, and this simplifies the factorization theorem.

First, it is well known that at large transverse momentum, the transverse spin asymmetry is higher twist, as I now review. In that region, we use distribution and fragmentation functions integrated over intrinsic transverse momentum. Now, in the absence of a measurement of the polarization of the outgoing hadron, the single-particle fragmentation is spin independent. On the other hand, the transverse-spin dependence of the distribution functions is only in the off-diagonal elements of quark density matrices [8]. Therefore we need the part of the hard scattering that is off-diagonal in the helicity of the initial state quark but diagonal in the (summed) final-state helicities. Helicity conservation at the vertices for the gluon, photon and $Z$ prohibits such a term, at leading twist.

But, at low transverse momentum, the fragmentation function has dependence on transverse spin — see eq. (4). The corresponding hard scattering is just elastic electron-quark scattering, and we need terms that are off-diagonal in the final state quark helicity. Quark helicity conservation requires that these terms are also off-diagonal in initial-state quark helicity, and we are therefore discussing a spin transfer from the incoming to the outgoing quark. The off-diagonal terms in the density matrix for the incoming quark are given by the transverse spin distribution defined in eq. (2).

Since there is a kinematic zero in the transverse spin dependence of the fragmentation at $k_{\perp}=0$, the spin dependence of the cross section must have transverse momentum dependence roughly proportional to the dimensionless coefficient

$$
\frac{q_{\perp} M}{q_{\perp}^{2}+M^{2}},
$$


where $M$ is a typical hadronic mass. This exhibits the kinematic zero when $q_{\perp}=0$, the leading twist asymmetry when $q_{\perp}=O(M)$, and the higher twist asymmetry when $q_{\perp} \gg M$.

We have now seen that the only transverse spin asymmetry at leading twist is in the low transverse momentum term in the generalized eq. (14). Moreover (5) implies that the transverse spin asymmetry has a $\sin \phi$ dependence on the azimuth $\phi$ of the transverse momentum, so that any kind of uniform azimuthal averaging will remove the asymmetry.

Following Meng, Olness and Soper [24] and earlier work, we could decompose the cross section in terms of scalar structure functions. In the case of fully inclusive unpolarized deeply inelastic scattering, with photon exchange, there are just two structure functions, the wellknown $F_{1}$ and $F_{2}$. But when we measure one of the particles, $B$, in the final state, there is an extra vector in the problem, so there are more structure functions, enumerated in [24]. When in addition we allow the incoming hadron to be polarized, there are extra structure functions, just as for the kinematically isomorphic Drell-Yan cross section [10,28]. We will choose instead to work directly with the cross section and its angular dependence. It would of course be of interest to perform a structure function analysis.

Since at leading twist, there is only transverse spin dependence in the low transverse momentum term in eq. (14), it is only this term that we need to change, with the result

$$
\begin{aligned}
E^{\prime} E_{B} \frac{d \sigma}{d^{3} l^{\prime} d^{3} p_{B}}= & \frac{4 x_{\mathrm{Bj}}}{Q^{2}} \sum_{a} \int d^{2} k_{a \perp} \hat{f}_{a / A}\left(x_{\mathrm{Bj}}, k_{a \perp}\right) \rho_{\alpha \alpha^{\prime}} \frac{d \hat{\sigma}_{\alpha \alpha^{\prime} ; \beta \beta^{\prime}}}{d \Omega} \hat{D}_{\beta \beta^{\prime} ; B / a}\left(z, k_{a \perp}+q_{\perp}\right) \\
& +Y\left(x_{\mathrm{Bj}}, Q, z, q_{\perp} / Q\right) .
\end{aligned}
$$

Temporarily we have changed notation: $\alpha, \alpha^{\prime}, \beta$ and $\beta^{\prime}$ represent indices for the initial and final-state densities matrices, $\rho_{\alpha \alpha^{\prime}}$ is the helicity density matrix for the incoming quark, while the indices $\beta \beta^{\prime}$ on the fragmentation function $\hat{D}$ represent the dependence of the fragmentation on the outgoing quark's density matrix.

We now recast this formula in terms of transverse spin vectors by using parity invariance as well as helicity conservation. First, we have already seen that helicity conservation tells us that the only transverse spin dependence is in the part of the hard scattering that is offdiagonal in helicity, i.e., $\hat{\sigma}_{+-;+-}$and its hermitian conjugate $\hat{\sigma}_{-+;-+}$. Thus the off-diagonal terms in the final and initial state density matrices are proportional: $\rho_{+-}^{\text {out }}=C \rho_{+-}$, where $C$ depends on the kinematic variables of the hard scattering. Furthermore, the expression for the off-diagonal term in a density matrix in terms of the spin vector is $\rho_{+-}=\frac{1}{2}\left|s_{\perp}\right| \mathrm{e}^{i \chi}$, apart from a possible phase and a possible sign error in the exponent. Here $\chi$ is the azimuthal angle of the transversity about the momentum of the quark. Furthermore parity invariance implies that if the spin of the initial quark is perpendicular to the plane of the scattering, then so is the spin of the final-state quark. Hence the coefficient $C$ is real, if we choose our conventions such that a real value of $\rho_{+-}$corresponds to a spin vector perpendicular to the plane of scattering.

It follows that the transversities of the initial and final quarks are proportional. The direction of the transversity of the final quark is obtained by rotating it about the normal to the plane of the hard scattering. (Fig. 3.)

Hence the spin dependence of low transverse momentum term in the cross section is given 


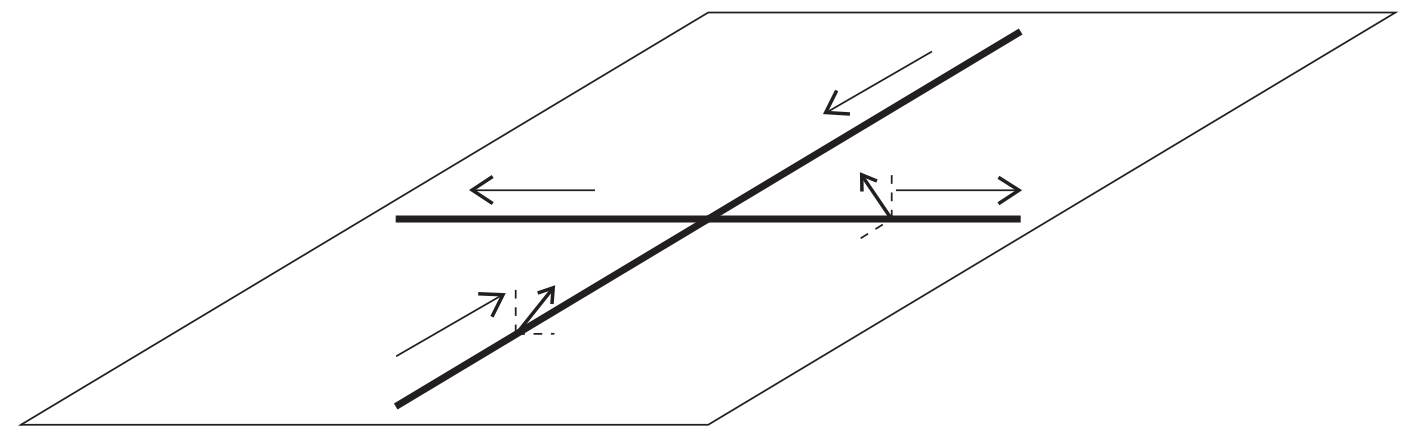

Fig. 3. Relation between spin vectors for initial and final state quarks. 
by the following factor:

$$
1+C \frac{\sum_{a} e_{a}^{2} \int d^{2} k_{a \perp} \Delta_{T} \hat{f} \Delta \hat{D}\left(s^{\prime}\right)}{\sum_{a} e_{a}^{2} \int d^{2} k_{a \perp} \hat{f} \hat{D}} .
$$

Here we have used the fact that the spin transfer coefficient $C$, which we will calculate in the next section, depends only on the scattering angle of the hard scattering. It is independent of quark flavor and the other kinematic variables; thus we can factor it out of the integrals. The sums are over flavors of quark and antiquark. The transversity vector $s^{\prime}$ that is used in the spin-dependent fragmentation function $\Delta \hat{D}\left(s^{\prime}\right)$ is the transverse spin vector of the initial hadron rotated in the center of mass of the hard scattering, according to the method of Fig. 3 .

The initial state distributions are functions of the length of $k_{a \perp}$ only. So after integration over $k_{a \perp}$, the cross section acquires a spin dependence whose dependence on the azimuth of $B$ is proportional to $\sin \phi$, where $\phi$ is the azimuthal angle of $p_{B}$, when measured in the lepton-parton center-of-mass about the outgoing jet axis (as defined by $p_{J}^{\mu}$ in eq. (10)), with $\phi=0$ being the direction of the vector $s^{\prime}$ defined just above.

\subsection{Calculation of SPin transfer}

We can easily compute the spin transfer part of the hard scattering cross section from the amplitudes given by Gastmans and $\mathrm{Wu}$ [29]. The cross section is for elastic electron-quark scattering, with all masses set to zero. Because of helicity conservation and parity invariance, there are two independent helicity amplitudes: $M_{++}$and $M_{+-}$, where the first and second indices label the helicities of the electron and quark. The amplitudes $M_{--}$and $M_{-+}$are related to these by parity invariance.

The unpolarized cross section is a standard kinematic factor times $\frac{1}{2}\left[\left|M_{++}\right|^{2}+\left|M_{+-}\right|^{2}\right]$. When the electron is unpolarized, the density matrix $\rho^{f}$ of the outgoing quark is expressed in terms of the density matrix $\rho^{i}$ of the incoming quark as follows:

$$
\rho^{f} \equiv\left(\begin{array}{cc}
\rho_{++}^{f} & \rho_{+-}^{f} \\
\rho_{-+}^{f} & \rho_{--}^{f}
\end{array}\right)=\left(\begin{array}{ll}
\rho_{++}^{i} & C \rho_{+-}^{i} \\
C \rho_{-+}^{i} & \rho_{--}^{i}
\end{array}\right),
$$

where

$$
C=\frac{M_{++} M_{+-}^{*}+M_{-+} M_{--}^{*}}{\left|M_{++}\right|^{2}+\left|M_{+-}\right|^{2}} .
$$

Up to a phase convention, the explicit values for the matrix elements are [29]:

$$
\begin{aligned}
& M_{++}=M_{--}=4 i e^{2} q_{a} \frac{1}{1-\cos \theta}, \\
& M_{-+}=M_{+-}=2 i e^{2} q_{a} \frac{1+\cos \theta}{1-\cos \theta} .
\end{aligned}
$$

Here $q_{a}$ is the electric charge of quark $a$ in units of the basic charge $e$, while $\theta$ is the scattering angle in the lepton-quark center-of-mass. The definitions of the helicity states are such that the scattering amplitude is purely imaginary; this convention differs from that of [29]. 
It follows that the spin transfer coefficient is

$$
\begin{aligned}
C & =\frac{4(1+\cos \theta)}{4+(1+\cos \theta)^{2}} \\
& =\frac{1-y}{1-y+\frac{1}{2} y^{2}},
\end{aligned}
$$

where $y$ is the usual variable $p_{A} \cdot\left(l-l^{\prime}\right) / p_{A} \cdot l$, which is $\left(E-E^{\prime}\right) / E$ in the target's rest frame and $(1-\cos \theta) / 2$ in the lepton-quark center-of-mass. The spin transfer coefficient is $100 \%$ at small scattering angles, and decreases to zero for exactly backward scattering. But even at $90^{\circ}(y=1 / 2)$, it is $80 \%$.

Note that the spin averaged square of the matrix element is

$$
\overline{|M|^{2}}=\frac{4 e^{4} q_{a}^{2}}{y^{2}}\left(1-y+\frac{1}{2} y^{2}\right)
$$

while the spin-dependent part is

$$
C \overline{|M|^{2}}=\frac{4 e^{4} q_{a}^{2}}{y^{2}}(1-y) .
$$

In the structure functions, the coefficient of $1-y$ feeds into $F_{2}$, while the coefficient of $y^{2} / 2$ feeds into $F_{1}$. Thus the transverse spin asymmetry we are discussing can be regarded as a $100 \%$ asymmetry in $F_{2}$ and a zero asymmetry in $F_{1}$.

\section{Predictions for DIS}

The measurements are of the single hadron distribution in the 'current quark fragmentation region' of collisions of unpolarized leptons on transversely polarized nucleons. An asymmetry of the cross section is expected under reversal of the nucleon polarization as a function of the azimuthal angle $\phi$ of the outgoing hadron around the jet axis, which is defined by parton model kinematics. The asymmetry will have a $\sin \phi$ dependence.

To a first approximation, the asymmetry is predicted to be the product of the polarization of the quarks in the initial nucleon, the spin transfer coefficient of the hard scattering, and the analyzing power of the fragmentation. In principle the asymmetry is a function of all the kinematic variables in the problem. The effect should be largest in the valence region for both the parton density and the fragmentation, say for $x_{\mathrm{Bj}} \gtrsim 0.3$ and $z \gtrsim 0.3$. One could even choose the hadron to be the leading hadron in the jet.

The data should be analyzed separately for different charges, and ideally for different flavors of hadron (e.g., kaon versus pion versus proton). For scattering on a proton target, the biggest asymmetry will probably be for $\pi^{+}$, since the $\pi^{+}$has a valence $u$ quark in common with the proton and deep inelastic scattering dominantly goes with $u$ quarks in the valence region. It is possible that the asymmetry will reverse sign for $\pi^{-}$, and the value for $\pi^{0}$ is not obvious. It would also be interesting to see how big the asymmetry is for $K^{+}$, which has a 
valence $u$ quark; this would probe the difference in the creation of strange quark-antiquark pairs in fragmentation as compared to creation of $u$ and $d$ pairs.

Furthermore there will be a strong dependence of the asymmetry on the transverse momentum $k_{\perp}$ of the hadron relative to the jet axis. This dependence will be roughly of the form of (15), up to an overall coefficient, and it will be important to verify this dependence. At small $k_{\perp}$, there is a kinematic zero, while, at large $k_{\perp}$, QCD predicts the asymmetry to be of higher twist. It is at $k_{\perp} \mathrm{s}$ of a few hundred $\mathrm{MeV}$ to about a $\mathrm{GeV}$ that one can expect the biggest asymmetry.

Note that the unpolarized cross section itself has a significant azimuthal dependence [30]. This is predicted [31] to be leading twist at large $k_{\perp}$, but higher twist at low $k_{\perp}$, unlike the behavior of the transverse spin asymmetry. But clearly this would be a confounding effect to be careful about in the experiments. One must measure the actual spin asymmetry in the cross section and not just the azimuthal dependence of the cross section.

Precise quantitative predictions cannot be made at present since the asymmetry depends on some not-yet-measured nonperturbative functions. Interesting comparisons can be made with data from proton-proton scattering and from final state correlations in $e^{+} e^{-}$annihilation. Ultimately we must wait for experiments to give us the values of the nonperturbative functions. They will probe the spin and chiral structure of both the initial hadron and of the fragmentation. The spin and chiral structure of fragmentation is at present a very little explored subject.

But we can suggest the general size, based on experience in other situations. The quarks in the hadron might be $50 \%$ polarized, as for the helicity asymmetry. The spin transfer in the hard scattering is mostly $80 \%$ to $100 \%$, from calculation. Finally, the fragmentation could have an analyzing power of tens of percent. (The last figure is the most uncertain!). Overall we can then reasonably expect about a $10 \%$ asymmetry in the cross section.

\section{$5 \quad$ Hadron-Hadron Scattering}

Exactly analogous measurements can be made in jet production in hadron-hadron scattering. To define the jet axis, one could measure the jet axis in a conventional manner; this would probably have too large an uncertainty. An example of a better way would be to measure the correlation between the leading particles in opposite jets. Intrinsic transverse momentum effects generate out-of-plane transverse momentum for these particles. The maximum asymmetry will be when the spin vector of the incoming polarized proton is in the plane of the hard scattering.

More theoretical analysis is needed here.

\section{Model Calculations of Fragmentation}

In this section I will present a very simple model calculation of the transversity dependent fragmentation function. It will show that the spin-dependence is permitted by the symme- 


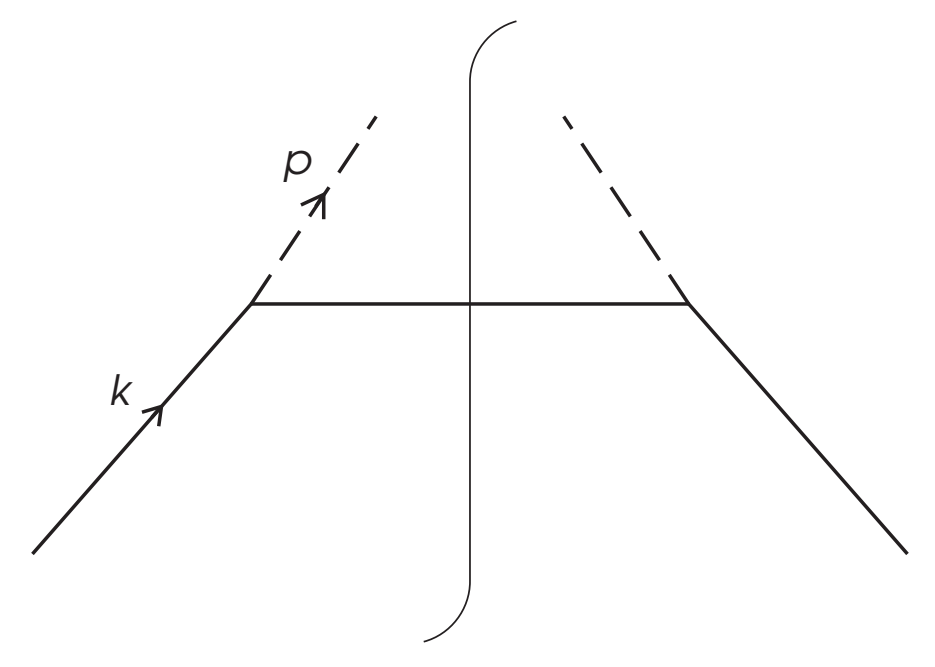

Fig. 4. Lowest order graph for fragmentation of quark to pion. The solid line is a quark and the dashed line is a pion.

tries of QCD, but that it requires non-trivial phases in the interaction. Furthermore, it will be clear that the transversity dependence is caused by the spontaneous breaking of chiral symmetry.

The model is a sigma model for pions coupled to quarks in the manner suggested by Georgi and Manohar [32]. The Lagrangian is to be considered an effective Lagrangian for suitable areas of nonperturbative QCD. While the model is not perfect, it does contain two important features of QCD: the quark degrees of freedom and the chiral symmetry breaking. We will calculate the lowest order graph for the fragmentation of a quark to a pion. To get a transversity dependence we will see that we must dress the propagators so that they acquire imaginary parts.

The graph is shown is Fig. 4, and its contribution to the unpolarized fragmentation 
follows from the definition eq. (3):

$$
\begin{aligned}
\hat{D}\left(z, k_{\perp}\right)=\frac{g^{2}}{16 \pi^{4}} \int d k^{-} \frac{1}{\left(k^{2}-M^{2}\right)^{2}} 2 \pi \delta^{(+)}\left((k-p)^{2}-M^{2}\right) \\
\frac{1}{4} \operatorname{tr} \gamma^{+}(\not k+M) \gamma_{5}(\not k-\not k+M) \gamma_{5}(\not k+M) .
\end{aligned}
$$

Here $g$ is the pion quark coupling, presumably around a third of $g_{\pi N N}$, for which $g_{\pi_{N N}}^{2} /\left(16 \pi^{2}\right) \approx 1.1$. The quark mass $M$ is due to chiral symmetry breaking, so it should be around $300 \mathrm{MeV}$. For simplicity, we will neglect the pion mass. Then a simple calculation gives

$$
\hat{D}=\frac{g^{2}}{16 \pi^{3}} \frac{1}{k_{\perp}^{2}+M^{2}},
$$

and results in a transverse momentum distribution with the canonical $300 \mathrm{MeV}$ width. The $1 / k_{\perp}^{2}$ tail at large $k_{\perp}$ is not to be trusted: it comes from a region where the quark is far off its mass shell, and there the sigma model is entirely inappropriate. Note the flavor structure: for different charge states in the fragmentation of a $u$-quark, we have $u \rightarrow \pi^{+}: u \rightarrow \pi^{0}: u \rightarrow \pi^{-}=2: 1: 0$.

Similarly, the transversity dependent fragmentation function (eq. (4)) in our model is

$$
\begin{aligned}
\Delta \hat{D}\left(z, k_{\perp}\right)=\frac{g^{2}}{16 \pi^{4}} \int d k^{-} & \frac{1}{\left(k^{2}-M^{2}\right)^{2}} 2 \pi \delta^{(+)}\left((k-p)^{2}-M^{2}\right) \\
& \frac{1}{4} \operatorname{tr} \gamma^{+} \gamma_{5} s_{\perp} \cdot \gamma_{\perp}(A \not k+B M) \gamma_{5}(\not k-\not k+M) \gamma_{5}\left(A^{*} \not k+B^{*} M\right) .
\end{aligned}
$$

Here, we have anticipated the need for imaginary parts in the amplitudes, and have used a dressed quark propagator $i(A \not k+B M) /\left(k^{2}-M^{2}\right)$, where the scalar coefficients $A$ and $B$ depend on $k^{2}$. On-shell renormalization gives them the value 1 when $k^{2}=M^{2}$. The result is

$$
\Delta \hat{D}=\frac{g^{2}}{16 \pi^{3}} \frac{2 M \operatorname{Im} A^{*} B}{\left(k_{\perp}^{2}+M^{2}\right)^{2}} \frac{(1-z)}{z}\left(s_{\perp}^{y} k^{x}-s_{\perp}^{x} k^{y}\right) .
$$

Hence the analyzing power is the ratio:

$$
\frac{\Delta \hat{D}}{\hat{D}}=\frac{2 M \operatorname{Im} A^{*} B}{k_{\perp}^{2}+M^{2}} \frac{(1-z)}{z}\left(s_{\perp}^{y} k^{x}-s_{\perp}^{x} k^{y}\right) .
$$

This exhibits the properties claimed earlier: It gives a $\sin \phi$ dependence on the azimuth of the transverse momentum, with a kinematic zero at $k_{\perp}=0$. A significant analyzing power evidently depends on the phases of $A$ and $B$ being substantially different, but there is presumably no reason why this should not be the case.

There is an apparent blow up of (28) when $z \rightarrow 0$. This is not actually the case, since the virtual quark then approaches its mass shell, where $A=B=1$. 


\section{Conclusions}

I have proposed that one can probe the transverse polarization of a quark that initiates a jet by measuring the azimuthal dependence of the hadrons in the jet, especially the leading hadrons. There are certainly other processes than the two explained in this paper that could be exploited. The typically large spin transfer in the hard scattering will aid in generating large asymmetries.

Perhaps the most interesting aspect of the idea is that it gives one a direct handle on how the chiral properties of the strong interactions couple to fragmentation. In particular, it would appear from the model calculations in Sec. 6 that the fragmentation asymmetry will only appear if there is breaking of chiral symmetry. Thus, in the context of fragmentation, it probes the known spontaneous breaking of chiral symmetry.

Conceivably, the measured asymmetry could be very small. This would require that the quarks in a transversely polarized proton have a small polarization or that the analyzing power of the fragmentation is small. The first possibility seems highly unlikely, given what we know of the other parton distributions in the valence region. The model calculation for the fragmentation indicates that there is no symmetry reason why the analyzing power should be small. Perhaps the best suggestion that a significant analyzing power is likely comes from the frequency of large spin asymmetries that are experimentally measured in hadron-induced processes.

The differences between the longitudinal and the transverse spin asymmetries in the distribution function are sensitive to relativistic and spin-orbit effects in the proton wave function, and are thus of intrinsic interest.

\section{Acknowledgments}

I would like to thank several colleagues for useful conversations, in particular Bob Carlitz, Steve Heppelmann, Bob Jaffe, Glenn Ladinsky, Lech Mankiewicz, Jian-Wei Qiu and George Sterman. This work was supported in part by the U.S. Department of Energy under grant DE-FG02-90ER-40577 and by the Texas National Laboratory Research Commission.

\section{Appendix}

Sivers [21] suggested that the $k_{\perp}$ distribution of a quark in a hadron could have an azimuthal asymmetry when the initial hadron has transverse polarization. However, as we will now show, such an asymmetry is prohibited because QCD is time-reversal invariant.

Consider

$$
\begin{aligned}
& \hat{f}_{a / A}\left(x, k_{\perp} ; \alpha, \alpha^{\prime}\right) \equiv \\
& \int \frac{\mathrm{d} y^{-} \mathrm{d}^{2} y_{\perp}}{(2 \pi)^{3}} \mathrm{e}^{-i x p^{+} y^{-}+i k_{\perp} \cdot y_{\perp}}\left\langle p \alpha\left|\bar{\psi}_{a}\left(0, y^{-}, y_{\perp}\right) \frac{\gamma^{+}}{2} \psi_{a}(0)\right| p \alpha^{\prime}\right\rangle,
\end{aligned}
$$


which is a matrix in the helicity indices $\alpha$ and $\alpha^{\prime}$. This quantity is the same as eq. (1), except that we have replaced the hadron states by particular spin states in a helicity basis. ( $\alpha$ and $\alpha^{\prime}$ take the values $L$ or $R$.) Contracting this matrix with the spin density matrix for the incoming hadron gives eq. (1).

It can readily be checked that the matrix $\hat{f}_{a / A}\left(x, k_{\perp} ; \alpha, \alpha^{\prime}\right)$ is hermitian, i.e.,

$$
\left[\hat{f}_{a / A}\left(x, k_{\perp} ; \alpha, \alpha^{\prime}\right)\right]^{\dagger} \equiv\left[\hat{f}_{a / A}\left(x, k_{\perp} ; \alpha^{\prime}, \alpha\right)\right]^{*}=\hat{f}_{a / A}\left(x, k_{\perp} ; \alpha, \alpha^{\prime}\right) .
$$

Furthermore, let us choose coordinates in which the incoming hadron has zero transverse momentum, $p_{\perp}=0$. Then invariance under rotations about the $z$ axis shows that the diagonal elements, $\hat{f}_{a / A}\left(x, k_{\perp} ; R R\right)$ and $\hat{f}_{a / A}\left(x, k_{\perp} ; L L\right)$ are functions of the length but not the angle of $k_{\perp}$. Parity invariance implies that these two diagonal elements are equal:

$$
\hat{f}_{a / A}\left(x, k_{\perp} ; R R\right)=\hat{f}_{a / A}\left(x, k_{\perp} ; L L\right) .
$$

We now apply a time-reversal transformation followed by a parity inversion. With the Bjorken and Drell [33] conventions for the Dirac matrices, we have

$$
(\mathcal{P} \mathcal{T})^{\dagger} \psi_{\alpha}(x) \mathcal{P} \mathcal{T}=\operatorname{PT} \psi(-x)
$$

with $P T=i \gamma^{0} \gamma^{1} \gamma^{3}$. Note that momenta are unchanged but helicity states get reversed under $\mathcal{P} \mathcal{T}$. Moreover there is a relative sign between the transformation of left and right-handed helicities:

$$
\begin{aligned}
\mathcal{P} \mathcal{T}|p, L\rangle & =|p, R\rangle, \\
\mathcal{P} \mathcal{T}|p, R\rangle & =-|p, L\rangle,
\end{aligned}
$$

where we have ignored an overall phase. The relative minus sign is essential, in order that $\mathcal{T}^{2}$ be -1 when acting on fermionic states. This sign can be verified from the transformation law of the Dirac field provided that one remembers that $\mathcal{T}$ is antilinear and that $T^{*}=-T$.

It follows that

$$
\begin{aligned}
& \hat{f}_{a / A}\left(x, k_{\perp} ; L R\right)=-\int \frac{\mathrm{d} y^{-} \mathrm{d}^{2} y_{\perp}}{(2 \pi)^{3}} \mathrm{e}^{-i x p^{+} y^{-}+i k_{\perp} \cdot y_{\perp}} \\
&\left\langle p, R\left|\mathcal{T}^{\dagger} \bar{\psi}_{a}\left(0, y^{-}, y_{\perp}\right) \mathcal{T} \frac{\gamma^{+}}{2} \mathcal{T}^{\dagger} \psi_{a}(0) \mathcal{T}\right| p, L\right\rangle^{*} \\
&=-\int \frac{\mathrm{d} y^{-} \mathrm{d}^{2} y_{\perp}}{(2 \pi)^{3}} \mathrm{e}^{-i x p^{+} y^{-}+i k_{\perp} \cdot y_{\perp}} \\
&\left\langle p, R\left|\bar{\psi}_{a}\left(0,-y^{-},-y_{\perp}\right) P T \frac{\gamma^{+}}{2} P T \psi_{a}(0)\right| p, L\right\rangle^{*} \\
&=-\hat{f}_{a / A}\left(x, k_{\perp} ; L R\right),
\end{aligned}
$$

where the complex conjugation arises because the time reversal operator $\mathcal{T}$ is antilinear, and the last line follows from the hermiticity of $\hat{f}\left(\alpha \alpha^{\prime}\right)$. It is now immediate that the off-diagonal elements are zero:

$$
\hat{f}(L R)=\hat{f}(R L)=0 .
$$


The matrix $\hat{f}\left(\alpha \alpha^{\prime}\right)$ is therefore proportional to the unit matrix, so that there is no dependence of the transverse momentum distribution on the spin of the incoming hadron. This contradicts Sivers' [Sivers] suggestion.

\section{References}

1 O. Nachtmann, Nucl. Phys. B127 (1977) 314.

2 R.H. Dalitz, G.R. Goldstein, and R. Marshall, Z. Phys. C 42 (1989) 441, and Phys. Lett. B215 (1988) 783; G.R. Goldstein, "Determining Quark Helicity from Jet Distributions" in Proc. of 8th Int. Symp. on High Energy Spin Physics, Minneapolis, MN, ed. K.J. Heller, AIP Conference Proceedings 187 (AIP, New York, 1989)

3 M.B. Einhorn, in "Spin Correlations in Quark and Gluon Fragmentation" in Proc. of 8th Int. Symp. on High Energy Spin Physics, Minneapolis, MN, ed. K.J. Heller, AIP Conference Proceedings 187 (AIP, New York, 1989).

4 A.V. Efremov, L. Mankiewicz and N.A. Törnqvist, Phys. Lett. B284 (1992) 394.

5 R. Carlitz, J.C. Collins, S. Heppelmann, R. Jaffe, X. Ji, G. Ladinsky, "Measuring Transversity Densities in Singly Polarized Hadron-Hadron Collisions", Penn State preprint $\mathrm{PSU} / \mathrm{TH} / 101$, in preparation.

6 European Muon Collaboration, M Arneodo et al., Nucl. Phys. B321 (1989) 541.

7 R.L. Jaffe and X.-D. Ji, Phys. Rev. Lett. 67 (1991) 552; X.-D. Ji, Phys. Lett. B284 (1992) 137.

8 X. Artru and M. Mekhfi, Z. Phys. C 45 (1990) 669.

9 K. Hidaka, E. Monsay and D. Sivers, Phys. Rev. D19 (1979) 1503.

10 J.P. Ralston and D.E. Soper, Nucl. Phys. B152 (1979) 109.

11 R. Carlitz and R. Willey, "Single Spin Asymmetries in Muon Pair Production" in Proceedings of the Polarized Collider Workshop, ed. J.C. Collins, S. Heppelmann and R. Robinett, AIP Conference Proceedings 223 (AIP, New York, 1991); R. Carlitz and R. Willey, "Determining Gluon And Anti-Quark Spin Fractions Via Single Spin Asymmetry Measurements", preprint PITT-91-11; R. Carlitz and R. Willey, Phys. Rev. D45 (1992) 2323.

12 E704 Collaboration: D.L. Adams et al., Phys. Lett. B264 (1991) 462, Phys. Lett. B261 (1991) 201, Phys. Lett. B276 (1992) 531, and references quoted there to earlier experiments at lower energies.

13 I. Balitsky and V. Braun, Nucl. Phys. B361 (1991) 93.

14 J.-W. Qiu and G. Sterman, Nucl. Phys. B353 (1991) 105, 137 and Phys. Rev. Lett. 67 (1991) 2264. 
15 J.C. Collins, D.E. Soper and G. Sterman, "Factorization of Hard Processes in QCD" in "Perturbative QCD" (A.H. Mueller, ed.) (World Scientific, Singapore, 1989), and references therein.

16 J.C. Collins, D.E. Soper and G. Sterman, Nucl. Phys. B261 (1975) 104 and B308 (1988) 833; G.T. Bodwin, Phys. Rev. D31 (1985) 2616 and D34 (1986) 3932.

17 J.C. Collins, D.E. Soper and G. Sterman, Nucl. Phys. B250 (1985) 199.

18 J.C. Collins and D.E. Soper, Nucl. Phys. B194 (1982) 445, and references therein.

19 J.C. Collins, Phys. Rev. D21 (1980) 2962.

20 D.E. Soper, Phys. Rev. D15 (1977) 1141; C. Bouchiat, P. Fayet and P. Meyer, Nucl. Phys. B34 (1971) 157.

21 D. Sivers, Phys. Rev. D41 (1990) 83 and D43 (1991) 261.

22 J.C. Collins, "Hard Scattering in QCD with Polarized Beams", Penn State preprint $\mathrm{PSU} / \mathrm{TH} / 100$.

23 S. Gasiorowicz, "Elementary Particle Physics" (Wiley, New York, 1966), page 515.

24 R. Meng, F. Olness, and D.E. Soper, Nucl. Phys. B371 (1992) 79.

25 R.P. Feynman, "Photon-Hadron Interactions" (Benjamin, Reading MA, 1972).

26 F.V. Tkachov, "Euclidean Asymptotic Expansions of Green Functions of Quantum Fields. (I) Expansions of Products of Singular Functions", preprint FERMILAB-PUB91/347-T, Int. J. Mod. Phys. A (1992) (in print) and refs. therein; G.B. Pivovarov and F.V. Tkachov: "Euclidean Asymptotic Expansions of Green Functions of Quantum Fields. (II) Combinatorics of the $A$ s-operation", preprint FERMILAB-PUB-91/345T; Int. J. Mod. Phys. A (1992) (in print).

27 J.C. Collins and D.E. Soper, Nucl. Phys. B193 (1981) 381.

28 J.T. Donohue and S. Gottlieb, Phys. Rev. D23 (1981) 2577,2581.

29 R. Gastmans and T.T. Wu, "The Ubiquitous Photon" (Oxford University Press, Oxford, 1990).

30 European Muon Collaboration, M Arneodo et al., Z. Phys. C 34 (1987) 277.

31 J. Chay, S.D. Ellis and W.J. Stirling, Phys. Rev. D45 (1992) 46.

32 A. Manohar and H. Georgi, Nucl. Phys. B234 (1984) 189.

33 J.D. Bjorken and S.D. Drell, "Relativistic Quantum Fields" (McGraw-Hill, New York, 1965). 